\title{
Positive solution of singular fractional differential system with nonlocal boundary conditions
}

Jing $\mathrm{Wu}^{1}$, Xinguang Zhang ${ }^{2,3^{*}}$, Lishan $\mathrm{Liu}^{4}$ and Yonghong $\mathrm{Wu}^{3}$

\section{*Correspondence:}

zxg123242@163.com

${ }^{2}$ School of Mathematical and

Informational Sciences, Yantai

University, Yantai, Shandong

264005, China

${ }^{3}$ Department of Mathematics and

Statistics, Curtin University of

Technology, Perth, 6845, Australia

Full list of author information is

available at the end of the article

\begin{abstract}
In this paper, we consider the existence of positive solutions for a singular fractional differential system involving a nonlocal boundary condition which is given by a linear functional on $C[0,1]$ with a signed measure. By looking for the upper and lower solutions of the system, the sufficient condition of the existence of positive solutions is established; some further cases are discussed. This is proved in the case of strong singularity and with a signed measure.
\end{abstract}

MSC: 34B15; 34B25

Keywords: singularity; fractional differential system; nonlocal boundary conditions; positive solution

\section{Introduction}

In this paper, we consider the existence of positive solutions for a singular nonlinear fractional differential system with nonlocal boundary conditions,

$$
\left\{\begin{array}{l}
-D_{0+x}^{\alpha} x(t)=f(t, x(t), y(t)), \quad-D_{0+}^{\beta} y(t)=g(t, x(t), y(t)), \quad 0<t<1, \\
x(0)=0, \quad x(1)=\int_{0}^{1} x(s) d A(s), \quad y(0)=0, \quad y(1)=\int_{0}^{1} y(s) d B(s),
\end{array}\right.
$$

where $1<\alpha, \beta \leq 2, D_{0+}^{\alpha}$ and $D_{0+}^{\beta}$ are the standard Riemann-Liouville derivatives, $\int_{0}^{1} x(s) d A(s)$ and $\int_{0}^{1} y(s) d B(s)$ denote the Riemann-Stieltjes integral, where $A, B$ are functions of bounded variation. $f, g:(0,1) \times(0,+\infty) \times(0,+\infty) \rightarrow[0,+\infty)$ are continuous and may be singular at $x=y=0$ and $t=0,1$.

In system (1.1), the boundary condition is given by a nonlocal condition involving a Stieltjes integral type linear functional on $C[0,1]$ with a signed measure, but it does not need to be a positive functional. In particular, if $d A(s)=d B(s)=d s$ or $h(s) d s$, then the BVP (1.1) reduces to an integral boundary value problem, and thus it also includes the multipoint boundary value problem as a special case. So the problem with Stieltjes integral boundary condition contains various boundary value problems (see [1]).

Since the nonlocal boundary value problems can describe a class of very interesting and important phenomena arising from heat conduction, chemical engineering, underground water flow, thermo-elasticity, and plasma physics, this type of problem has attracted much attention of many researchers (see [2-14] and the references therein). Especially, based

(02014 Wu et al.; licensee Springer. This is an Open Access article distributed under the terms of the Creative Commons Attribution License (http://creativecommons.org/licenses/by/2.0), which permits unrestricted use, distribution, and reproduction in any medium, provided the original work is properly cited. 
on the fixed point theory of a strict set of contraction operators in a cone, Feng et al. [5] investigated the existence and nonexistence of positive solutions of the following second order BVPs with integral boundary conditions in Banach space:

$$
\left\{\begin{array}{l}
u^{\prime \prime}(t)+f(t, u)=\theta, \quad t \in(0,1), \\
u(0)=\int_{0}^{1} g(t) u(t) d t, \quad u(1)=\theta, \text { or } \\
u(0)=\theta, \quad u(1)=\int_{0}^{1} g(t) u(t) d t .
\end{array}\right.
$$

Subsequently, Liu et al. [6] studied a singular integral boundary value problem,

$$
\left\{\begin{array}{l}
u^{\prime \prime}(t)+a(t) u^{\prime}(t)+b(t) u(t)+c(t) f(u)=0, \quad t \in(0,1) \\
u(0)=\int_{0}^{1} g(s) u(s) d s, \quad u(1)=\int_{0}^{1} h(s) u(s) d s
\end{array}\right.
$$

where $a \in C[0,1], b \in C([0,1],(-\infty, 0)), c \in C((0,1),[0,+\infty)), f \in C((0,+\infty),[0,+\infty))$, and $g, h \in L^{1}[0,1]$ are nonnegative. $c(t) \not \equiv 0$ is allowed to be singular at $t=0,1$, and $f$ may be singular at $u=0$. By using the fixed point index theorem, the existence of positive solutions for the BVP (1.3) is established.

By means of a monotone iterative technique, Zhang and Han [4] established the existence and uniqueness of the positive solutions for a class of higher conjugate-type fractional differential equation with one nonlocal term,

$$
\left\{\begin{array}{l}
D_{0+}^{\alpha} x(t)+f(t, x(t))=0, \quad 0<t<1, n-1<\alpha \leq n \\
x^{(k)}(0)=0, \quad 0 \leq k \leq n-2, \quad x(1)=\int_{0}^{1} x(s) d A(s),
\end{array}\right.
$$

where $\alpha \geq 2, D_{0_{+}}^{\alpha}$ is the standard Riemann-Liouville derivative, $A$ is a function of bounded variation, $\int_{0}^{1} u(s) d A(s)$ denotes the Riemann-Stieltjes integral of $u$ with respect to $A, d A$ can be a signed measure. Recently, some work on systems of nonlinear fractional differential equations was developed [7-9]. In [7], Ahmad and Ntouyas studied the existence and uniqueness of solutions for a system of Hadamard type fractional differential equations with integral boundary conditions

$$
\begin{cases}{ }^{c} D^{\alpha} u(t)=f(t, u(t), v(t)), & 1<t<e, 1<\alpha \leq 2, \\ { }^{c} D^{\beta} u(t)=g(t, u(t), v(t)), & 1<t<e, 1<\beta \leq 2, \\ u(1)=0, \quad u(e)=I^{\gamma} u\left(\sigma_{1}\right)=\frac{1}{\Gamma(\gamma)} \int_{1}^{\sigma_{1}}\left(\log \frac{\sigma_{1}}{s}\right)^{\gamma-1} \frac{u(s)}{s} d s & v^{\sigma_{2}}\left(\log \frac{\sigma_{2}}{s}\right)^{\gamma-1} \frac{v(s)}{s} d s\end{cases}
$$

where $\gamma>0,1<\sigma_{1}<e, 1<\sigma_{2}<e, D^{(\cdot)}$ is the Hadamard fractional derivative of fractional order, $I^{\gamma}$ is the Hadamard fractional integral of order $\gamma$ and $f, g:[1, e] \times R \times R \rightarrow R$ are continuous functions. The existence of solutions for the system (1.4) is derived from LeraySchauder's alternative, whereas the uniqueness of the solution is established by the Banach contraction principle. More recently, Ahmad et al. [8] studied the existence of solutions for a system of coupled hybrid fractional differential equations with Dirichlet boundary conditions. By using the standard tools of the fixed point theory, the existence and uniqueness results were established.

Motivated by the above work, we consider the existence of positive solutions for the singular fractional differential system with nonlocal Stieltjes integral boundary conditions 
when $f, g$ can be singular at $t=0,1$ and $x=y=0$. It is well known from linear elastic fracture mechanics that the stress near the crack tip exhibits a power singularity of $r^{-0.5}$ [1], where $r$ is the distance measured from the crack tip, and this classical singularity also exists in nonlocal nonlinear problems. But due to the singularity of $f, g$ at $x=y=0$, we cannot handle the system (1.1) like in $[4,5]$. Thus, this work we shall devote to finding the upper and lower solution of the system (1.1), and by means of the Schauder fixed point theorem to establish the criterion of the existence of positive solutions for the system (1.1). To the best of our knowledge, there has been no work done for the singular fractional differential system with the Riemann-Stieltjes integral boundary conditions, and this work aims to contribute in this field. Our work also extends the results of $[4-6,9]$ to fractional systems with which $f, g$ can be singular at $t=0,1$ and $x=y=0$.

\section{Preliminaries and lemmas}

The basic space used in this paper is $E=C([0,1] ; \mathbb{R}) \times C([0,1] ; \mathbb{R})$, where $\mathbb{R}$ is a real number set. Obviously, the space $E$ is a Banach space if it is endowed with the norm as follows:

$$
\|(x, y)\|:=\|x\|+\|y\|, \quad\|x\|=\max _{t \in[0,1]}|x(t)|, \quad\|y\|=\max _{t \in[0,1]}|y(t)|
$$

for any $(x, y) \in E$. By a positive solution of problem (1.1), we mean a pair of functions $(u, v) \in$ $E$ satisfying (1.1) with $u(t) \geq 0, v(t) \geq 0$ for all $t \in[0,1]$ and $(u, v) \neq(0,0)$.

Now we begin our work based on theory of fractional calculus; for details of the definitions and semigroup properties of Riemann-Liouville fractional calculus, one refers to [15-17]. In what follows, we give the definitions of the lower and upper solution of the system (1.1).

Definition 2.1 A pair of functions $\left(\phi_{1}(t), \psi_{1}(t)\right) \in E$ is called a lower solution of the system (1.1), if it satisfies

$$
\left\{\begin{array}{l}
-D_{0+}^{\alpha} \phi_{1}(t) \leq f\left(t, \phi_{1}(t), \psi_{1}(t)\right), \quad-D_{0+}^{\beta} \psi_{1}(t) \leq g\left(t, \phi_{1}(t), \psi_{1}(t)\right), \quad 0<t<1, \\
\phi_{1}(0) \geq 0, \quad \phi_{1}(1) \geq \int_{0}^{1} \phi_{1}(s) d A(s), \quad \psi_{1}(0) \geq 0, \quad \psi_{1}(1) \geq \int_{0}^{1} \psi_{1}(s) d B(s) .
\end{array}\right.
$$

Definition 2.2 A pair of functions $\left(\phi_{2}(t), \psi_{2}(t)\right) \in E$ is called an upper solution of the system (1.1), if it satisfies

$$
\left\{\begin{array}{l}
-D_{0+}^{\alpha} \phi_{2}(t) \geq f\left(t, \phi_{2}(t), \psi_{2}(t)\right), \quad-D_{0+}^{\beta} \psi_{2}(t) \geq g\left(t, \phi_{2}(t), \psi_{2}(t)\right), \quad 0<t<1, \\
\phi_{2}(0) \leq 0, \quad \phi_{2}(1) \leq \int_{0}^{1} \phi_{2}(s) d A(s), \quad \psi_{2}(0) \leq 0, \quad \psi_{2}(1) \leq \int_{0}^{1} \psi_{2}(s) d B(s) .
\end{array}\right.
$$

Remark 2.1 Normally, it is difficult to find the lower solution and upper solution of the system (1.1). In Theorem 3.1 of this paper, we will give a general strategy to find the lower solution and upper solution of the system (1.1) through a series of integral calculations form the initial value $\left(t^{\alpha-1}, t^{\beta-1}\right)$.

Next let

$$
G_{\alpha}(t, s)=\frac{1}{\Gamma(\alpha)} \begin{cases}{[t(1-s)]^{\alpha-1},} & 0 \leq t \leq s \leq 1, \\ {[t(1-s)]^{\alpha-1}-(t-s)^{\alpha-1},} & 0 \leq s \leq t \leq 1,\end{cases}
$$




$$
G_{\beta}(t, s)=\frac{1}{\Gamma(\beta)} \begin{cases}{[t(1-s)]^{\beta-1},} & 0 \leq t \leq s \leq 1 \\ {[t(1-s)]^{\beta-1}-(t-s)^{\beta-1},} & 0 \leq s \leq t \leq 1\end{cases}
$$

and define

$$
\mathcal{G}_{A}(s)=\int_{0}^{1} G_{\alpha}(t, s) d A(t), \quad \mathcal{G}_{B}(s)=\int_{0}^{1} G_{\beta}(t, s) d B(t) .
$$

According to the strategy of [4], we can get easily the Green functions of the corresponding linear boundary value problem for the system (1.1).

Lemma 2.1 Given $h \in L^{1}(0,1)$ and $1<\alpha, \beta \leq 2$, then the following boundary value problems:

$$
\left\{\begin{array} { l } 
{ - D _ { 0 + } ^ { \alpha } x ( t ) = h ( t ) , \quad 0 < t < 1 , } \\
{ x ( 0 ) = 0 , \quad x ( 1 ) = \int _ { 0 } ^ { 1 } x ( s ) d A ( s ) , }
\end{array} \quad \left\{\begin{array}{l}
-D_{0+}^{\beta} y(t)=h(t), \quad 0<t<1, \\
y(0)=0, \quad y(1)=\int_{0}^{1} y(s) d B(s),
\end{array}\right.\right.
$$

have the unique solution

$$
x(t)=\int_{0}^{1} H_{\alpha}(t, s) h(s) d s, \quad y(t)=\int_{0}^{1} H_{\beta}(t, s) h(s) d s,
$$

where $H_{\alpha}(t, s), H_{\beta}(t, s)$ are the Green functions of the BVP (2.4), respectively, and

$$
H_{\alpha}(t, s)=\frac{t^{\alpha-1}}{1-\mathcal{A}} \mathcal{G}_{A}(s)+G_{\alpha}(t, s), \quad H_{\beta}(t, s)=\frac{t^{\beta-1}}{1-\mathcal{B}} \mathcal{G}_{B}(s)+G_{\beta}(t, s),
$$

where

$$
\mathcal{A}=\int_{0}^{1} t^{\alpha-1} d A(t), \quad \mathcal{B}=\int_{0}^{1} t^{\beta-1} d B(t)
$$

Lemma 2.2 Let $0 \leq \mathcal{A}, \mathcal{B}<1$ and $\mathcal{G}_{A}(s), \mathcal{G}_{B}(s) \geq 0$ for $s \in[0,1]$, then the Green functions defined by (2.6) satisfy

(1) $H_{\alpha}(t, s), H_{\beta}(t, s)>0$, for all $t, s \in(0,1)$.

(2) There exist two constants $\lambda$, $\mu$ such that

$$
\begin{aligned}
& \frac{t^{\alpha-1}}{1-\mathcal{A}} \mathcal{G}_{A}(s) \leq H_{\alpha}(t, s) \leq \lambda t^{\alpha-1}, \\
& \frac{t^{\beta-1}}{1-\mathcal{B}} \mathcal{G}_{B}(s) \leq H_{\beta}(t, s) \leq \mu t^{\beta-1}, \quad s, t \in[0,1] .
\end{aligned}
$$

Proof (1) is obvious. We only prove the first inequality of (2.7), the proof of second one is similar to those of the first one.

Since $G_{\alpha}(t, s) \geq 0$ for any $s, t \in[0,1]$, we have

$$
\frac{t^{\alpha-1}}{1-\mathcal{A}} \mathcal{G}_{A}(s) \leq H_{\alpha}(t, s), \quad s, t \in[0,1] .
$$

On the other hand, from (2.1), obviously,

$$
G_{\alpha}(t, s) \leq \frac{1}{\Gamma(\alpha)} t^{\alpha-1}
$$


Take

$$
\lambda=\frac{1}{\Gamma(\alpha)}+\frac{\max _{0 \leq s \leq 1} \mathcal{G}_{A}(s)}{1-\mathcal{A}},
$$

then we have

$$
H_{\alpha}(t, s) \leq \lambda t^{\alpha-1}
$$

The proof is completed.

Lemmas 2.1 and 2.2 lead to the following maximum principle.

Lemma 2.3 If $(x, y) \in E$ satisfies

$$
x(0)=0, \quad x(1)=\int_{0}^{1} x(s) d A(s), \quad y(0)=0, \quad y(1)=\int_{0}^{1} y(s) d B(s),
$$

and $D_{0+}^{\alpha} x(t) \leq 0, D_{0+}^{\alpha} y(t) \leq 0$ for any $t \in(0,1)$. Then

$$
x(t) \geq 0, \quad y(t) \geq 0, \quad t \in[0,1] .
$$

Lemma 2.4 (Schauder fixed point theorem) Let $T$ be a continuous and compact mapping of a Banach space E into itself, such that the set

$$
\{x \in E: x=\sigma T x, \text { for some } 0 \leq \sigma \leq 1\}
$$

is bounded. Then T has a fixed point.

\section{Main results}

We make the following assumptions throughout this paper:

(H0) $A$ and $B$ are functions of bounded variation satisfying $\mathcal{G}_{A}(s), \mathcal{G}_{B}(s) \geq 0$ for $s \in[0,1]$ and $0 \leq \mathcal{A}, \mathcal{B}<1$

(H1) $f, g \in C((0,1) \times(0,+\infty) \times(0, \infty),[0,+\infty))$ are decreasing in second and third variables and such that

$$
f\left(s, s^{\alpha-1}, s^{\beta-1}\right), g\left(s, s^{\alpha-1}, s^{\beta-1}\right) \in L^{1}(0,1) ;
$$

(H2) for all $r \in(0,1)$, there exist constants $0<\epsilon, \sigma<1$ such that, for any $(t, x, y) \in(0,1) \times(0,+\infty) \times(0,+\infty)$

$$
f(t, r x, r y) \leq r^{-\epsilon} f(t, x, y), \quad g(t, r x, r y) \leq r^{-\sigma} g(t, x, y) .
$$

Remark 3.1 The conditions (H1)-(H2) imply that $f, g$ have a powder singularity at $x=y=0$, and some typical functions are $\sum_{i=1}^{m}\left(x^{-\lambda_{i}}+y^{-\mu_{i}}\right), 0<\lambda_{i}<1,0<\mu_{i}<1$ with $\epsilon=\min _{1 \leq i \leq m}\left\{\lambda_{i}, \mu_{i}\right\}$ and $\lambda_{i}(\alpha-1)<1, \mu_{i}(\beta-1)<1, i=1,2, \ldots, m$. 
Theorem 3.1 Suppose (H0)-(H2) hold. Then the system (1.1) has at least a positive solution $\left(x^{*}, y^{*}\right)$, which satisfies

$$
\left(L^{-1} t^{\alpha-1}, L^{-1} t^{\beta-1}\right) \leq\left(x^{*}, y^{*}\right) \leq\left(L t^{\alpha-1}, L t^{\beta-1}\right)
$$

where

$$
\begin{aligned}
L= & \max \left\{\left[\lambda \int_{0}^{1} f\left(s, s^{\alpha-1}(1-s)\right) d s\right]^{\frac{1}{1-\epsilon}},\left[\mu \int_{0}^{1} f\left(s, s^{\alpha-1}(1-s)\right) d s\right]^{\frac{1}{1-\sigma}},\right. \\
& {\left.\left[\frac{1-\mathcal{A}}{\int_{0}^{1} \mathcal{G}_{A}(s) f\left(s, s^{\alpha-1}, s^{\beta-1}\right) d s}\right]^{\frac{1}{1-\epsilon}},\left[\frac{1-\mathcal{B}}{\int_{0}^{1} \mathcal{G}_{B}(s) g\left(s, s^{\alpha-1}, s^{\beta-1}\right) d s}\right]^{\frac{1}{1-\sigma}}, 1\right\} . }
\end{aligned}
$$

In particular, if $L=1$, then $\left(t^{\alpha-1}, t^{\beta-1}\right)$ is positive solution of the system (1.1).

Proof Define a cone

$$
P=\left\{(x, y) \in E: L^{-1} t^{\alpha-1} \leq x(t) \leq L t^{\alpha-1}, L^{-1} t^{\beta-1} \leq y(t) \leq L t^{\beta-1}, t \in[0,1]\right\},
$$

then $P$ is nonempty since $\left(t^{\alpha-1}, t^{\beta-1}\right) \in P$. Now let us denote an operator $T$ by

$$
T(x, y)(t)=\left(T_{1}(x, y)(t), T_{2}(x, y)(t)\right), \quad \text { for any }(x, y) \in P,
$$

where

$$
T_{1}(x, y)(t)=\int_{0}^{1} H_{\alpha}(t, s) f(s, x(s), y(s)) d s, \quad T_{2}(x, y)(t)=\int_{0}^{1} H_{\beta}(t, s) g(s, x(s), y(s)) d s .
$$

We claim that $T$ is well defined and $T(P) \subset P$.

In fact, for any $(x, y) \in P$, we have

$$
L^{-1} t^{\alpha-1} \leq x(t) \leq L t^{\alpha-1}, \quad L^{-1} t^{\beta-1} \leq y(t) \leq L t^{\beta-1}, \quad t \in[0,1] .
$$

So from Lemma 2.1 and (H1)-(H2), one gets

$$
\begin{aligned}
T_{1}(x, y)(t) & =\int_{0}^{1} H_{\alpha}(t, s) f(s, x(s), y(s)) d s \\
& \leq \int_{0}^{1} \lambda t^{\alpha-1} f\left(s, L^{-1} s^{\alpha-1}, L^{-1} s^{\beta-1}\right) d s \\
& \leq \lambda L^{\epsilon} t^{\alpha-1} \int_{0}^{1} f\left(s, s^{\alpha-1}, s^{\beta-1}\right) d s \leq L t^{\alpha-1}
\end{aligned}
$$

and

$$
\begin{aligned}
T_{2}(x, y)(t) & =\int_{0}^{1} H_{\beta}(t, s) g(s, x(s), y(s)) d s \\
& \leq \int_{0}^{1} \mu t^{\beta-1} g\left(s, L^{-1} s^{\alpha-1}, L^{-1} s^{\beta-1}\right) d s \\
& \leq \mu L^{\sigma} t^{\beta-1} \int_{0}^{1} g\left(s, s^{\alpha-1}, s^{\beta-1}\right) d s \leq L t^{\beta-1} .
\end{aligned}
$$


On the other hand, by Lemma 2.1 and (H1)-(H2), we also have

$$
\begin{aligned}
T_{1}(x, y)(t) & \geq \frac{t^{\alpha-1}}{1-\mathcal{A}} \int_{0}^{1} \mathcal{G}_{A}(s) f\left(s, L s^{\alpha-1}, L s^{\beta-1}\right) d s \\
& \geq \frac{L^{-\epsilon} t^{\alpha-1}}{1-\mathcal{A}} \int_{0}^{1} \mathcal{G}_{A}(s) f\left(s, s^{\alpha-1}, s^{\beta-1}\right) d s \\
& \geq L^{-\epsilon} t^{\alpha-1} \geq L^{-1} t^{\alpha-1},
\end{aligned}
$$

and

$$
\begin{aligned}
T_{2}(x, y)(t) & \geq \frac{t^{\beta-1}}{1-\mathcal{B}} \int_{0}^{1} \mathcal{G}_{B}(s) g\left(s, L s^{\alpha-1}, L s^{\beta-1}\right) d s \\
& \geq \frac{L^{-\sigma} t^{\beta-1}}{1-\mathcal{B}} \int_{0}^{1} \mathcal{G}_{B}(s) g\left(s, s^{\alpha-1}, s^{\beta-1}\right) d s \\
& \geq L^{-\sigma} t^{\beta-1} \geq L^{-1} t^{\beta-1} .
\end{aligned}
$$

Thus it follows from (3.3)-(3.6) that $T$ is well defined and $T(P) \subset P$. Moreover, by Lemma 2.2, we have

$$
\left\{\begin{array}{l}
-D_{0+}^{\alpha} T_{1}(x, y)(t)=f\left(t, T_{1}(x, y)(t), T_{2}(x, y)(t)\right) \\
-D_{0+}^{\beta} T_{2}(x, y)(t)=g\left(t, T_{1}(x, y)(t), T_{2}(x, y)(t)\right) \\
T_{1}(x, y)(t)(0)=0, \quad T_{1}(x, y)(1)=\int_{0}^{1} T_{1}(x, y)(s) d A(s) \\
T_{2}(x, y)(0)=0, \quad T_{2}(x, y)(1)=\int_{0}^{1} T_{2}(x, y)(s) d B(s) .
\end{array}\right.
$$

Now take

$$
\begin{array}{ll}
\underline{\varphi}(t)=\min \left\{t^{\alpha-1}, T_{1}\left(t^{\alpha-1}, t^{\beta-1}\right)\right\}, & \bar{\varphi}(t)=\max \left\{t^{\alpha-1}, T_{1}\left(t^{\alpha-1}, t^{\beta-1}\right)\right\}, \\
\underline{\psi}(t)=\min \left\{t^{\beta-1}, T_{2}\left(t^{\alpha-1}, t^{\beta-1}\right)\right\}, & \bar{\psi}(t)=\max \left\{t^{\beta-1}, T_{2}\left(t^{\alpha-1}, t^{\beta-1}\right)\right\},
\end{array}
$$

since $\left(t^{\alpha-1}, t^{\beta-1}\right) \in P,\left(T_{1}\left(t^{\alpha-1}, t^{\beta-1}\right), T_{2}\left(t^{\alpha-1}, t^{\beta-1}\right)\right) \in P$, we have

$$
(\underline{\varphi}, \underline{\psi}) \in P, \quad(\bar{\varphi}, \bar{\psi}) \in P, \quad \text { and } \quad \underline{\varphi} \leq t^{\alpha-1} \leq \bar{\varphi}, \quad \underline{\psi} \leq t^{\beta-1} \leq \bar{\psi} .
$$

Let

$$
\left(\varphi_{1}, \psi_{1}\right)=\left(T_{1}(\underline{\varphi}, \underline{\psi}), T_{2}(\underline{\varphi}, \underline{\psi})\right), \quad\left(\varphi_{2}, \psi_{2}\right)=\left(T_{1}(\bar{\varphi}, \bar{\psi}), T_{2}(\bar{\varphi}, \bar{\psi})\right)
$$

then by (3.8)-(3.11) and (H2), we have

$$
\begin{aligned}
\left(\varphi_{2}, \psi_{2}\right) & =\left(T_{1}(\bar{\varphi}, \bar{\psi}), T_{2}(\bar{\varphi}, \bar{\psi})\right) \leq\left(T_{1}\left(t^{\alpha-1}, t^{\beta-1}\right), T_{2}\left(t^{\alpha-1}, t^{\beta-1}\right)\right) \\
& \leq\left(T_{1}(\underline{\varphi}, \underline{\psi}), T_{2}(\underline{\varphi}, \underline{\psi})\right)=\left(\varphi_{1}, \psi_{1}\right), \\
\left(\varphi_{2}, \psi_{2}\right) & \leq\left(T_{1}\left(t^{\alpha-1}, t^{\beta-1}\right), T_{2}\left(t^{\alpha-1}, t^{\beta-1}\right)\right) \leq(\bar{\varphi}, \bar{\psi}), \\
\left(\varphi_{1}, \psi_{1}\right) & \geq\left(T_{1}\left(t^{\alpha-1}, t^{\beta-1}\right), T_{2}\left(t^{\alpha-1}, t^{\beta-1}\right)\right) \geq(\underline{\varphi}, \underline{\psi}) .
\end{aligned}
$$


Consequently, it follows from (3.7) and (3.10)-(3.13) that

$$
\begin{aligned}
& D_{0+}^{\alpha} \varphi_{1}(t)+f\left(t, \varphi_{1}(t), \psi_{1}(t)\right) \\
& =D_{0+}^{\alpha} T_{1}(\underline{\varphi}, \underline{\psi})(t)+f\left(t, \varphi_{1}(t), \psi_{1}(t)\right) \\
& =-f(t, \underline{\varphi}(t), \underline{\psi}(t))+f\left(t, \varphi_{1}(t), \psi_{1}(t)\right) \\
& \leq-f(t, \underline{\varphi}(t), \underline{\psi}(t))+f(t, \underline{\varphi}(t), \underline{\psi}(t))=0, \\
& \varphi_{1}(0)=0, \quad \varphi_{1}(1)=\int_{0}^{1} \varphi_{1}(s) d A(s), \\
& D_{0+}^{\beta} \psi_{1}(t)+g\left(t, \varphi_{1}(t), \psi_{1}(t)\right) \\
& =D_{0+}^{\beta} T_{2}\left(\underline{\varphi}, \underline{\psi}(t)+g\left(t, \varphi_{1}(t), \psi_{1}(t)\right)\right. \\
& =-g(t, \underline{\varphi}(t), \underline{\psi}(t))+g\left(t, \varphi_{1}(t), \psi_{1}(t)\right) \\
& \leq-g(t, \underline{\varphi}(t), \underline{\psi}(t))+g(t, \underline{\varphi}(t), \underline{\psi}(t))=0, \\
& \psi_{1}(0)=0, \quad \psi_{1}(1)=\int_{0}^{1} \psi_{1}(s) d B(s),
\end{aligned}
$$

and

$$
\begin{gathered}
D_{0+}^{\alpha} \varphi_{2}(t)+f\left(t, \varphi_{2}(t), \psi_{2}(t)\right) \\
=D_{0+}^{\alpha} T_{1}(\bar{\varphi}, \bar{\psi})(t)+f\left(t, \varphi_{2}(t), \psi_{2}(t)\right) \\
=-f(t, \bar{\varphi}(t), \bar{\psi}(t))+f\left(t, \varphi_{2}(t), \psi_{2}(t)\right) \\
\geq-f(t, \bar{\varphi}(t), \bar{\psi}(t))+f(t, \bar{\varphi}(t), \bar{\psi}(t))=0, \\
\varphi_{2}(0)=0, \quad \varphi_{2}(1)=\int_{0}^{1} \varphi_{2}(s) d A(s), \\
D_{0+}^{\beta} \psi_{2}(t)+g\left(t, \varphi_{2}(t), \psi_{2}(t)\right) \\
=D_{0+}^{\beta} T_{2}(\bar{\varphi}, \bar{\psi})(t)+g\left(t, \varphi_{2}(t), \psi_{2}(t)\right) \\
=-g(t, \bar{\varphi}(t), \bar{\psi}(t))+g\left(t, \varphi_{2}(t), \psi_{2}(t)\right) \\
\geq-g(t, \bar{\varphi}(t), \bar{\psi}(t))+g(t, \bar{\varphi}(t), \bar{\psi}(t))=0, \\
\psi_{2}(0)=0, \quad \psi_{2}(1)=\int_{0}^{1} \psi_{2}(s) d B(s), \\
D_{0+}^{\beta} \psi_{1}(t)+g\left(t, \varphi_{1}(t), \psi_{1}(t)\right) \\
=D_{0+}^{\beta} T_{2}(\underline{\varphi}, \underline{\psi})(t)+g\left(t, \varphi_{1}(t), \psi_{1}(t)\right) \\
=-g(t, \underline{\varphi}(t), \underline{\psi}(t))+g\left(t, \varphi_{1}(t), \psi_{1}(t)\right) \\
\leq-g(t, \underline{\varphi}(t), \underline{\psi}(t))+g(t, \underline{\varphi}(t), \underline{\psi}(t))=0, \\
\psi_{1}(0)=0, \quad \psi_{1}(1)=\int_{0}^{1} \psi_{1}(s) d B(s) .
\end{gathered}
$$

It follows from (3.12) and (3.14)-(3.18) that $\left(\varphi_{2}, \psi_{2}\right),\left(\varphi_{1}, \psi_{1}\right)$ are lower and upper solutions of the system (1.1), and $\left(\varphi_{2}, \psi_{2}\right),\left(\varphi_{1}, \psi_{1}\right) \in P$. 
Define the functions $\widetilde{F}, \widetilde{G}$, and the operator $\widetilde{T}$ in $E$ by

$$
\begin{aligned}
& \widetilde{F}(t, x, y)= \begin{cases}f\left(t, \varphi_{2}(t), \psi_{2}(t)\right), & (x, y)<\left(\varphi_{2}, \psi_{2}\right), \\
f(t, x, y), & \left(\varphi_{2}, \psi_{2}\right) \leq(x, y) \leq\left(\varphi_{1}, \psi_{1}\right), \\
f\left(t, \varphi_{1}(t), \psi_{1}(t)\right), & (x, y)>\left(\varphi_{1}, \psi_{1}\right),\end{cases} \\
& \widetilde{G}(t, x, y)= \begin{cases}g\left(t, \varphi_{2}(t), \psi_{2}(t)\right), & (x, y)<\left(\varphi_{2}, \psi_{2}\right), \\
g(t, x, y), & \left(\varphi_{2}, \psi_{2}\right) \leq(x, y) \leq\left(\varphi_{1}, \psi_{1}\right), \\
g\left(t, \varphi_{1}(t), \psi_{1}(t)\right), & (x, y)>\left(\varphi_{1}, \psi_{1}\right),\end{cases}
\end{aligned}
$$

and $\widetilde{T}=\left(\widetilde{T}_{1}(x, y)(t), \widetilde{T}_{2}(x, y)(t)\right)$ where

$$
\widetilde{T}_{1}(x, y)(t)=\int_{0}^{1} H_{\alpha}(t, s) \widetilde{F}(s, x(s), y(s)) d s, \quad \widetilde{T}_{2}(x, y)(t)=\int_{0}^{1} H_{\beta}(t, s) \widetilde{G}(s, x(s), y(s)) d s .
$$

It follows from the assumption that $\widetilde{F}:(0,1) \times[0,+\infty) \times[0,+\infty) \rightarrow[0,+\infty)$ and $\widetilde{G}$ : $(0,1) \times[0,+\infty) \times[0,+\infty) \rightarrow[0,+\infty)$ are continuous. Consider the following boundary value problem:

$$
\left\{\begin{array}{l}
-D_{0+}^{\alpha} x(t)=\widetilde{F}(t, x(t), y(t)), \quad-D_{0+}^{\beta} y(t)=\widetilde{G}(t, x(t), y(t)), \quad 0<t<1, \\
x(0)=0, \quad x(1)=\int_{0}^{1} x(s) d A(s), \quad y(0)=0, \quad y(1)=\int_{0}^{1} y(s) d B(s) .
\end{array}\right.
$$

Obviously, a fixed point of the operator $\widetilde{T}$ is a solution of the BVP (3.21).

For all $(x, y) \in E$, by (3.19)-(3.20), we have

$$
\begin{aligned}
\widetilde{T}_{1}(x, y)(t) & \leq \int_{0}^{1} \lambda \widetilde{F}(s, x(s), y(s)) d s \leq \lambda \int_{0}^{1} f\left(s, \varphi_{2}(s), \psi_{2}(s)\right) d s \\
& \leq \lambda \int_{0}^{1} f\left(s, L^{-1} s^{\alpha-1}, L^{-1} s^{\beta-1}\right) d s \\
& \leq \lambda L^{\epsilon} \int_{0}^{1} f\left(s, s^{\alpha-1}, s^{\beta-1}\right) d s<+\infty \\
\widetilde{T}_{2}(x, y)(t) & \leq \int_{0}^{1} \mu \widetilde{G}(s, x(s), y(s)) d s \leq \mu \int_{0}^{1} g\left(s, L^{-1} s^{\alpha-1}, L^{-1} s^{\beta-1}\right) d s \\
& \leq \mu L^{\sigma} \int_{0}^{1} g\left(s, s^{\alpha-1}, s^{\beta-1}\right) d s<+\infty .
\end{aligned}
$$

So $\|\widetilde{T}\|=\left\|\widetilde{T}_{1}\right\|+\left\|\widetilde{T}_{2}\right\|<+\infty$, which implies that $\widetilde{T}$ is uniformly bounded. In addition, it follows from the continuity of $\widetilde{F}, \widetilde{G}$ and the uniform continuity of $H_{\alpha}, H_{\beta}$, and (H1) that $\widetilde{T}: E \times E \rightarrow E$ is continuous.

Let $\Omega \subset E \times E$ be bounded, by standard discuss and the Arzela-Ascoli theorem, we easily know $\widetilde{T}(\Omega)$ is equicontinuous. Thus $\widetilde{T}: E \rightarrow E$ is completely continuous, and by using Schauder fixed point theorem, $\widetilde{T}$ has at least a fixed point $\left(x^{*}, y^{*}\right)$ such that $\left(x^{*}, y^{*}\right)=\widetilde{T}\left(x^{*}, y^{*}\right)$.

Now we prove

$$
\left(\varphi_{2}(t), \psi_{2}(t)\right) \leq\left(x^{*}, y^{*}\right) \leq\left(\varphi_{1}(t), \psi_{1}(t)\right), \quad t \in[0,1] .
$$


We firstly prove $\left(x^{*}, y^{*}\right) \leq\left(\varphi_{1}(t), \psi_{1}(t)\right)$. Otherwise, suppose $\left(x^{*}, y^{*}\right)>\left(\varphi_{1}(t), \psi_{1}(t)\right)$. According to the definition of $\widetilde{F}, \widetilde{G}$, we have

$$
\begin{aligned}
& -D_{0+}^{\alpha} x^{*}(t)=\widetilde{F}\left(t, x^{*}(t), y^{*}(t)\right)=f\left(t, \varphi_{1}(t), \psi_{1}(t)\right), \\
& -D_{0+}^{\beta} y^{*}(t)=\widetilde{G}\left(t, x^{*}(t), y^{*}(t)\right)=g\left(t, \varphi_{1}(t), \psi_{1}(t)\right) .
\end{aligned}
$$

On the other hand, as $\left(\varphi_{1}(t), \psi_{1}(t)\right)$ is an upper solution of (1.1), we have

$$
-D_{0+}^{\alpha} \varphi_{1}(t) \geq f\left(t, \varphi_{1}(t), \psi_{1}(t)\right), \quad-D_{0+}^{\beta} \psi_{1}(t) \geq g\left(t, \varphi_{1}(t), \psi_{1}(t)\right) .
$$

Let $z(t)=\varphi_{1}(t)-x^{*}(t), w(t)=\psi_{1}(t)-y^{*}(t),(3.23)-(3.24)$ imply that

$$
D_{0+}^{\alpha} z(t)=D_{0_{+}}^{\alpha} \varphi_{1}(t)-D_{0_{+}}^{\alpha} x^{*}(t) \leq 0, \quad D_{0+}^{\beta} w(t)=D_{0+}^{\beta} \psi_{1}(t)-D_{0+}^{\alpha} y^{*}(t) \leq 0 .
$$

On the other hand, since $\left(\varphi_{1}(t), \psi_{1}(t)\right)$ is an upper solution of the BVP $(1.1)$ and $\left(x^{*}, y^{*}\right)$ is a fixed point of $\widetilde{T}$, we know

$$
z(0)=0, \quad z(1)=\int_{0}^{1} z(s) d A(s), \quad w(0)=0, \quad w(1)=\int_{0}^{1} w(s) d B(s) .
$$

It follows from Lemma 2.3 that

$$
z(t) \geq 0, \quad w(t) \geq 0
$$

i.e., $\left(x^{*}(t), y^{*}(t)\right) \leq\left(\varphi_{1}(t), \psi_{1}(t)\right)$ on $[0,1]$, which contradicts $\left(x^{*}, y^{*}\right)>\left(\varphi_{1}(t), \psi_{1}(t)\right)$. Thus we have $\left(x^{*}(t), y^{*}(t)\right) \leq\left(\varphi_{1}(t), \psi_{1}(t)\right)$ on $[0,1]$. In the same way, $\left(x^{*}(t), y^{*}(t)\right) \geq\left(\varphi_{2}(t), \psi_{2}(t)\right)$ on $[0,1]$. Consequently, $(3.22)$ is satisfied; then $\left(x^{*}(t), y^{*}(t)\right)$ is a positive solution of the problem (1.1)

It follows from $\left(\varphi_{2}(t), \psi_{2}(t)\right),\left(\varphi_{1}(t), \psi_{1}(t)\right) \in P$ and (3.22) that

$$
\left(L^{-1} t^{\alpha-1}, L^{-1} t^{\beta-1}\right) \leq\left(x^{*}, y^{*}\right) \leq\left(L t^{\alpha-1}, L t^{\beta-1}\right) .
$$

The proof is completed.

\section{Further results}

In this section, we discuss some special case for system (1.1) and obtain some further results. We firstly discuss that $f, g$ have no singularity at $x, y=0$, but can be singular at $t=0,1$.

Theorem 4.1 Suppose (H0) holds, and $f$, $g$ satisfies

( $\left.\mathrm{H}^{*} 1\right) f, g \in C((0,1) \times[0, \infty) \times[0, \infty),[0,+\infty))$ are decreasing in second and third variables and such that

$$
0<\int_{0}^{1} f(s, 0,0) d s<\infty, \quad 0<\int_{0}^{1} g(s, 0,0) d s<\infty .
$$


Then the system (1.1) has at least a positive solution $\left(x^{*}, y^{*}\right)$, which satisfies

$$
(0,0) \leq\left(x^{*}, y^{*}\right) \leq\left(\widetilde{L} t^{\alpha-1}, \widetilde{L} t^{\beta-1}\right)
$$

where

$$
\widetilde{L}=\max \left\{\lambda \int_{0}^{1} f(s, 0,0) d s, \mu \int_{0}^{1} g(s, 0,0) d s\right\} .
$$

Proof Similar to the proof of Theorem 3.1, we take the cone

$$
P_{1}=\{(x, y) \in E: x(t) \geq 0, y(t) \geq 0, t \in[0,1]\} .
$$

Clearly, $T\left(P_{1}\right) \subseteq P_{1}$ is well defined.

Now take

$$
\begin{array}{ll}
\underline{\varphi}(t)=0, \quad \bar{\varphi}(t)=T_{1}(0,0), \\
\underline{\psi}(t)=0, \quad \bar{\psi}(t)=T_{2}(0,0) ;
\end{array}
$$

we have

$$
(\underline{\varphi}, \underline{\psi}) \in P_{1}, \quad(\bar{\varphi}, \bar{\psi}) \in P_{1}, \quad \text { and } \quad \underline{\varphi}=0 \leq \bar{\varphi}, \quad \underline{\psi}=0 \leq \bar{\psi} .
$$

Let

$$
\left(\varphi_{1}, \psi_{1}\right)=\left(T_{1}(\underline{\varphi}, \underline{\psi}), T_{2}(\underline{\varphi}, \underline{\psi})\right), \quad\left(\varphi_{2}, \psi_{2}\right)=\left(T_{1}(\bar{\varphi}, \bar{\psi}), T_{2}(\bar{\varphi}, \bar{\psi})\right),
$$

then by (4.1)-(4.4) and $\left(\mathrm{H}^{*} 1\right)$, we have

$$
\begin{aligned}
\left(\varphi_{2}, \psi_{2}\right) & =\left(T_{1}(\bar{\varphi}, \bar{\psi}), T_{2}(\bar{\varphi}, \bar{\psi})\right) \leq\left(T_{1}(0,0), T_{2}(0,0)\right) \\
& \leq\left(T_{1}(\underline{\varphi}, \underline{\psi}), T_{2}(\underline{\varphi}, \underline{\psi})\right)=\left(\varphi_{1}, \psi_{1}\right), \\
\left(\varphi_{2}, \psi_{2}\right) & \leq\left(T_{1}(0,0), T_{2}(0,0)\right)=(\bar{\varphi}, \bar{\psi}), \\
\left(\varphi_{1}, \psi_{1}\right) & =\left(T_{1}(0,0), T_{2}(0,0)\right) \geq(\underline{\varphi}, \underline{\psi}) .
\end{aligned}
$$

Consequently, it follows from (4.5) and (4.6) that

$$
\begin{aligned}
D_{0+}^{\alpha} & \varphi_{1}(t)+f\left(t, \varphi_{1}(t), \psi_{1}(t)\right) \\
\quad & =D_{0+}^{\alpha} T_{1}(\underline{\varphi}, \underline{\psi})(t)+f\left(t, \varphi_{1}(t), \psi_{1}(t)\right) \\
& =-f(t, 0,0)+f\left(t, \varphi_{1}(t), \psi_{1}(t)\right) \leq-f(t, 0,0)+f(t, 0,0)=0, \\
D_{0+}^{\beta} & \psi_{1}(t)+g\left(t, \varphi_{1}(t), \psi_{1}(t)\right) \\
& =D_{0+}^{\beta} T_{2}(\underline{\varphi}, \underline{\psi})(t)+g\left(t, \varphi_{1}(t), \psi_{1}(t)\right) \\
& =-g(t, 0,0)+g\left(t, \varphi_{1}(t), \psi_{1}(t)\right) \leq-g(t, 0,0)+g(t, 0,0)=0,
\end{aligned}
$$


and

$$
\begin{aligned}
& D_{0+}^{\alpha} \varphi_{2}(t)+f\left(t, \varphi_{2}(t), \psi_{2}(t)\right) \\
& \quad=D_{0+}^{\alpha} T_{1}(\bar{\varphi}, \bar{\psi})(t)+f\left(t, \varphi_{2}(t), \psi_{2}(t)\right) \\
& \quad=-f(t, \bar{\varphi}(t), \bar{\psi}(t))+f\left(t, \varphi_{2}(t), \psi_{2}(t)\right) \\
& \quad \geq-f(t, \bar{\varphi}(t), \bar{\psi}(t))+f(t, \bar{\varphi}(t), \bar{\psi}(t))=0, \\
& D_{0+}^{\beta} \psi_{2}(t)+g\left(t, \varphi_{2}(t), \psi_{2}(t)\right) \\
& \quad=D_{0+}^{\beta} T_{2}(\bar{\varphi}, \bar{\psi})(t)+g\left(t, \varphi_{2}(t), \psi_{2}(t)\right) \\
& \quad=-g(t, \bar{\varphi}(t), \bar{\psi}(t))+g\left(t, \varphi_{2}(t), \psi_{2}(t)\right) \\
& \quad \geq-g(t, \bar{\varphi}(t), \bar{\psi}(t))+g(t, \bar{\varphi}(t), \bar{\psi}(t))=0 .
\end{aligned}
$$

Thus (4.5) and (4.7)-(4.10) imply that $\left(\varphi_{2}, \psi_{2}\right),\left(\varphi_{1}, \psi_{1}\right)$ are lower and upper solutions of the system $(1.1)$, and $\left(\varphi_{2}, \psi_{2}\right),\left(\varphi_{1}, \psi_{1}\right) \in P_{1}$.

On the other hand, by Lemma 2.2 ,

$$
\begin{aligned}
& \varphi_{1}(t)=T_{1}(0,0)(t)=\int_{0}^{1} H_{\alpha}(t, s) f(s, 0,0) d s \leq \lambda t^{\alpha-1} \int_{0}^{1} f(s, 0,0) d s \leq \widetilde{L} t^{\alpha-1}, \\
& \psi_{1}(t)=T_{2}(0,0)(t)=\int_{0}^{1} H_{\beta}(t, s) g(s, 0,0) d s \leq \mu t^{\beta-1} \int_{0}^{1} g(s, 0,0) d s \leq \widetilde{L} t^{\beta-1} .
\end{aligned}
$$

Thus the rest of proof is similar to those of Theorem 3.1.

Next, if $f, g$ have no singularity at $x, y=0$ and $t=0,1$, we copy the proof of Theorem 4.1, and we have the following interesting result.

Theorem 4.2 Suppose (H0) holds, $f(t, 0,0) \not \equiv 0, g(t, 0,0) \not \equiv 0, t \in[0,1]$, and $f, g \in$ $C([0,1] \times[0, \infty) \times[0, \infty),[0,+\infty))$ are decreasing in second and third variables. Then the system (1.1) has at least a positive solution $\left(x^{*}, y^{*}\right)$, which satisfies

$$
(0,0) \leq\left(x^{*}, y^{*}\right) \leq\left(\widetilde{L} t^{\alpha-1}, \widetilde{L} t^{\beta-1}\right)
$$

where

$$
\widetilde{L}=\max \left\{\lambda \int_{0}^{1} f(s, 0,0) d s, \mu \int_{0}^{1} g(s, 0,0) d s\right\} .
$$

\section{Examples}

Take functions of bounded variation,

$$
A(t)=\left\{\begin{array}{ll}
0, & t \in\left[0, \frac{1}{3}\right), \\
\frac{1}{2}, & t \in\left[\frac{1}{3}, \frac{2}{3}\right), \\
1, & t \in\left[\frac{2}{3}, 1\right],
\end{array} \quad B(t)= \begin{cases}0, & t \in\left[0, \frac{1}{2}\right), \\
2, & t \in\left[\frac{1}{2}, \frac{3}{4}\right), \\
1, & t \in\left[\frac{3}{4}, 1\right] .\end{cases}\right.
$$


Example 5.1 Suppose that $\alpha_{i}, \beta_{i}, \gamma_{i}>0$, and $0<\gamma_{i}+\frac{1}{2} \alpha_{i}<1,0<\gamma_{i}+\frac{1}{3} \beta_{i}<1, i=1,2$. We consider the following singular fractional differential system:

$$
-D_{0+}^{\frac{3}{2}} x(t)=t^{-\gamma_{1}}\left(x^{-\alpha_{1}}+y^{-\beta_{1}}\right), \quad-D_{0+}^{\frac{4}{3}} y(t)=t^{-\gamma_{2}}\left(x^{-\alpha_{2}}+y^{-\beta_{2}}\right),
$$

subject to the nonlocal boundary condition

$$
x(0)=0, \quad x(1)=\int_{0}^{1} x(s) d A(s), \quad y(0)=0, \quad y(1)=\int_{0}^{1} y(s) d B(s) .
$$

By a simple calculation, the system (5.1) with boundary condition (5.2) is equivalent to the following system with coefficients of both signs:

$$
\left\{\begin{array}{l}
-D_{0+}^{\frac{3}{2}} x(t)=t^{-\gamma_{1}}\left(x^{-\alpha_{1}}+y^{-\beta_{1}}\right), \quad-D_{0+}^{\frac{4}{3}} y(t)=t^{-\gamma_{2}}\left(x^{-\alpha_{2}}+y^{-\beta_{2}}\right), \\
x(0)=0, \quad x(1)=\frac{1}{2} x\left(\frac{2}{3}\right)+\frac{1}{2} x\left(\frac{1}{3}\right), \quad y(0)=0, \quad y(1)=2 y\left(\frac{1}{2}\right)-y\left(\frac{3}{4}\right),
\end{array}\right.
$$

and

$$
\begin{aligned}
& 0 \leq \mathcal{A}=\int_{0}^{1} t^{\frac{1}{2}} d A(t)=\frac{1}{2}\left(\frac{2}{3}\right)^{\frac{1}{2}}+\frac{1}{2}\left(\frac{1}{3}\right)^{\frac{1}{2}} \approx 0.6969<1, \\
& 0 \leq \mathcal{B}=\int_{0}^{1} t^{\frac{1}{3}} d B(t)=2\left(\frac{1}{2}\right)^{\frac{1}{3}}-\left(\frac{3}{4}\right)^{\frac{1}{3}} \approx 0.6788<1 .
\end{aligned}
$$

Clearly, $\mathcal{G}_{A}(s), \mathcal{G}_{B}(s) \geq 0$ for $s \in[0,1]$ also hold.

Let $f(t, x, y)=t^{-\gamma_{1}}\left(x^{-\alpha_{1}}+y^{-\beta_{1}}\right), g(t, x, y)=t^{-\gamma_{2}}\left(x^{-\alpha_{2}}+y^{-\beta_{2}}\right)$, then $f, g$ are decreasing in $x$ and $y$, and

$$
f\left(s, s^{\alpha-1}, s^{\beta-1}\right)=s^{-\gamma_{1}-\frac{1}{2} \alpha_{1}}+s^{-\gamma_{1}-\frac{1}{3} \beta_{1}}, g\left(s, s^{\alpha-1}, s^{\beta-1}\right)=s^{-\gamma_{2}-\frac{1}{2} \alpha_{2}}+s^{-\gamma_{2}-\frac{1}{3} \beta_{2}} \in L^{1}(0,1) .
$$

Moreover, for all $r \in(0,1)$ and $(t, x, y) \in(0,1) \times(0,+\infty) \times(0,+\infty)$, we have

$$
f(t, r x, r y) \leq r^{-\max \left\{\alpha_{1}, \beta_{1}\right\}} f(t, x, y), \quad g(t, r x, r y) \leq r^{-\max \left\{\alpha_{2}, \beta_{2}\right\}} g(t, x, y) .
$$

By Theorem 3.1, the system (5.1) with boundary condition (5.2) has at least a positive solution $\left(x^{*}, y^{*}\right)$.

Example 5.2 Consider the singular fractional differential system

$$
-D_{0+}^{\frac{3}{2}} x(t)=t^{-\frac{1}{4}}\left(\frac{1}{x^{2}+2}+\cos y\right), \quad-D_{0+}^{\frac{4}{3}} y(t)=\frac{|\ln t|+t^{-\frac{1}{2}}}{\left(x^{\frac{1}{2}}+1\right)(\sin y+1)},
$$

subject to nonlocal boundary condition (5.2). Let

$$
f(t, x, y)=t^{-\frac{1}{4}}\left(\frac{1}{x^{2}+2}+\cos y\right), \quad g(t, x, y)=\frac{|\ln t|+t^{-\frac{1}{2}}}{\left(x^{\frac{1}{2}}+1\right)(\sin y+1)},
$$


then $f, g$ are decreasing in $x$ and $y$, and

$$
\int_{0}^{1} f(s, 0,0) d s=\frac{3}{2} \int_{0}^{1} s^{-\frac{1}{4}} d s=2, \quad \int_{0}^{1} g(s, 0,0) d s=\int_{0}^{1}\left(s^{-\frac{1}{2}}-\ln s\right) d s=2 .
$$

Thus by Theorem 4.1, the system (5.3) with boundary condition (5.2) has at least a positive solution $\left(x^{*}, y^{*}\right)$.

Remark 5.1 In this work, the monotone assumption of $f$ and $g$ is an essential condition. In particular, for nonsingular case, the result is interesting since only monotone assumption is requested, which meets a large classes of functions.

\section{Competing interests}

The authors declare that they have no competing interests.

\section{Authors' contributions}

The work presented here was carried out in collaboration between all authors. JW and XZ completed the main part of this paper, LL and YW corrected the main theorems and polished the manuscript. All authors read and approved the final manuscript.

\section{Author details}

${ }^{1}$ School of Economic Mathematics, Southwestern University of Finance and Economics, Chengdu, Sichuan 610074, China. ${ }^{2}$ School of Mathematical and Informational Sciences, Yantai University, Yantai, Shandong 264005, China. ${ }^{3}$ Department of Mathematics and Statistics, Curtin University of Technology, Perth, 6845, Australia. ${ }^{4}$ School of Mathematical Sciences, Qufu Normal University, Qufu, Shandong 273165, China.

\section{Acknowledgements}

The authors were supported financially by the National Natural Science Foundation of China (11371221).

Received: 20 August 2014 Accepted: 4 December 2014 Published: 22 Dec 2014

\section{References}

1. Webb, JRL, Infante, G: Positive solutions of nonlocal boundary value problems: a unified approach. J. Lond. Math. Soc. 74, 673-693 (2006)

2. Gallardo, JM: Second order differential operators with integral boundary conditions and generation of semigroups. Rocky Mt. J. Math. 30, 1265-1292 (2000)

3. Lomtatidze, A, Malaguti, L: On a nonlocal boundary-value problems for second order nonlinear singular differential equations. Georgian Math. J. 7, 133-154 (2000)

4. Zhang, $X$, Han, Y: Existence and uniqueness of positive solutions for higher order nonlocal fractional differential equations. Appl. Math. Lett. 25, 555-560 (2012)

5. Feng, $M$, Ji, D, Ge, W: Positive solutions for a class of boundary-value problem with integral boundary conditions in Banach spaces. J. Comput. Appl. Math. 222, 351-363 (2008)

6. Liu, L, Hao, X, Wu, Y: Positive solutions for singular second order differential equations with integral boundary conditions. Math. Comput. Model. 57(3-4), 836-847 (2013)

7. Ahmad, B, Ntouyas, S: A fully Hadamard type integral boundary value problem of a coupled system of fractional differential equations. Fract. Calc. Appl. Anal. 17(2), 348-360 (2014)

8. Ahmad, B, Ntouyas, S, Alsaedi, A: Existence results for a system of coupled hybrid fractional differential equations. Sci. World J. 2014, Article ID 426438 (2014)

9. Henderson, J, Luca, R: Positive solutions for a system of fractional differential equations with coupled integral boundary conditions. Appl. Math. Comput. 249, 182-197 (2014)

10. Zhang, $X, L i u, L, W u, Y$ : Existence results for multiple positive solutions of nonlinear higher order perturbed fractional differential equations with derivatives. Appl. Math. Comput. 219, 1420-1433 (2012)

11. Zhang, $X, L i u, L, W u, Y$ : Multiple positive solutions of a singular fractional differential equation with negatively perturbed term. Math. Comput. Model. 55, 1263-1274 (2012)

12. Zhang, $X, \mathrm{Liu}, \mathrm{L}, \mathrm{Wu}, \mathrm{Y}$ : The eigenvalue problem for a singular higher order fractional differential equation involving fractional derivatives. Appl. Math. Comput. 218, 8526-8536 (2012)

13. Zhang, $X, L i u, L, W u, Y$ : The uniqueness of positive solution for a fractional order model of turbulent flow in a porous medium. Appl. Math. Lett. 37, 26-33 (2014)

14. Zhang, X, Liu, L, Wiwatanapataphee, $B$, Wu, Y: The eigenvalue for a class of singular $p$-Laplacian fractional differential equations involving the Riemann-Stieltjes integral boundary condition. Appl. Math. Comput. 235, 412-422 (2014)

15. Podlubny, I: Fractional Differential Equations, Mathematics in Science and Engineering. Academic Press, New York (1999)

16. Miller, K, Ross, B: An Introduction to the Fractional Calculus and Fractional Differential Equations. Wiley, New York (1993)

17. Kilbas, A, Srivastava, H, Trujillo, J: Theory and Applications of Fractional Differential Equations. Elsevier, Amsterdam (2006) 
10.1186/1687-1847-2014-323

Cite this article as: Wu et al.: Positive solution of singular fractional differential system with nonlocal boundary conditions. Advances in Difference Equations 2014, 2014:323

Submit your manuscript to a SpringerOpen ${ }^{\circ}$ journal and benefit from:

- Convenient online submission

- Rigorous peer review

- Immediate publication on acceptance

Open access: articles freely available online

- High visibility within the field

- Retaining the copyright to your article 